Research Article

\title{
On the Low-Degree Solution of the Sylvester Matrix Polynomial Equation
}

\author{
Yunbo Tian ${ }^{1}$ and Chao $\mathrm{Xia}^{2}$ \\ ${ }^{1}$ School of Mathematics and Statistics, Linyi University, Linyi, Shandong 276000, China \\ ${ }^{2}$ School of Mathematics and Statistics, Changchun University of Technology, Changchun, China
}

Correspondence should be addressed to Yunbo Tian; tianyunbobo@163.com

Received 13 June 2021; Accepted 25 July 2021; Published 31 July 2021

Academic Editor: Fazlollah Soleymani

Copyright (c) 2021 Yunbo Tian and Chao Xia. This is an open access article distributed under the Creative Commons Attribution License, which permits unrestricted use, distribution, and reproduction in any medium, provided the original work is properly cited.

We study the low-degree solution of the Sylvester matrix equation $\left(A_{1} \lambda+A_{0}\right) X(\lambda)+Y(\lambda)\left(B_{1} \lambda+B_{0}\right)=C_{0}$, where $A_{1} \lambda+A_{0}$ and $B_{1} \lambda+B_{0}$ are regular. Using the substitution of parameter variables $\lambda$, we assume that the matrices $A_{0}$ and $B_{0}$ are invertible. Thus, we prove that if the equation is solvable, then it has a low-degree solution $(L(\lambda), M(\lambda))$, satisfying the degree conditions $\delta L(\lambda)<\operatorname{Ind}\left(A_{0}^{-1} A_{1}\right)$ and $\delta M(\lambda)<\operatorname{Ind}\left(B_{1} B_{0}^{-1}\right)$.

\section{Introduction}

Dealing with the problems about regulation output in control theory leads to a generalized Sylvester matrix equation:

$$
A X+Y B=C
$$

where the matrices involved are the matrix polynomials (e.g., [1-7]). There have been an extensive study and application of the generalized Sylvester matrix equation (e.g., [8-11]). This work investigates the bound of the low-degree solution of equation (1) with degree 1 matrix polynomials $A$ and $B$.

We adopt the following terminology [12]. Let $H(\lambda)=$ $\sum_{i=0}^{l} H_{i} \lambda^{i}$ with $H_{l} \neq 0$, and we denote the degree of matrix polynomial $H(\lambda)$ by $\delta(H)=l$. If $H(\lambda)=0$, we set the degree $\delta(H)=-\infty$. A matrix polynomial $H(\lambda)$ is called regular if $\operatorname{det} H(\lambda) \neq 0$ and monic if $H_{l}=I$ is an identity matrix.

Wimmer used Jordan chains of polynomial to characterize the solvability of the generalized Sylvester equation in [13]. This condition about solvability extends results of Kučera [14] and Gohberg and Lerer [15]. In [16], Barnett studied the existence and uniqueness of the low-degree solutions. For monic matrices $A, B$, and $\delta(C) \leq m+n-1$, he proved that equation (1) has a unique solution satisfying
$\delta(X)<n, \delta(Y)<m$ if and only if the determinants of $A(\lambda)$ and $B(\lambda)$ are coprime. Feinstein and Bar-Ness [17] extended this result to the case with only $A(\lambda)$ or $B(\lambda)$ (not necessarily both) being regular. The solvability of matrix equation (1) was also studied in $[18,19]$.

It is well known that there are polynomials $u(\lambda)$ and $v(\lambda)$, such that

$$
u(\lambda) f(\lambda)+v(\lambda) g(\lambda)=\operatorname{gcd}(f(\lambda), g(\lambda))
$$

and $\delta u<\delta g, \delta v<\delta f$, where $\operatorname{gcd}(f(\lambda), g(\lambda))$ is the monic greatest common factor of $f(\lambda), g(\lambda)$. When we consider the case in Sylvester matrix polynomial equation (1), it is shown that for monic matrix polynomials $A(\lambda)$ and $B(\lambda)$, if equation (1) has solutions, then it has a solution $(X, Y)$ satisfying $\delta X<\delta B, \delta Y<\delta A$. However, the remark in [20] shows that the proposition is false when $A(\lambda)$ and $B(\lambda)$ are not monic matrix polynomials. As equation (1) with regular matrix polynomials $A$ and $B$ have not been developed fully about the degree, we will investigate the low-degree solution of

$$
\left(A_{1} \lambda+A_{0}\right) X(\lambda)+Y(\lambda)\left(B_{1} \lambda+B_{0}\right)=C_{0},
$$

where $A_{1} \lambda+A_{0}$ and $B_{1} \lambda+B_{0}$ are regular. We use the index of matrix to characterize the bound of low-degree solution. 


\section{Preliminary}

To prove Theorem 2, we first recall the division of matrix polynomials [12]. We restrict ourselves to the case when the dividend is a general matrix polynomial:

$$
H(\lambda)=\sum_{i=0}^{l} H_{i} \lambda^{i},
$$

and the divisor is a monic matrix polynomial:

$$
X(\lambda)=\sum_{i=0}^{m-1} X_{i} \lambda^{i}+I \lambda^{m} .
$$

In this case, we have the following representation:

$$
H(\lambda)=Q_{r}(\lambda) X(\lambda)+R_{r}(\lambda)
$$

where $Q_{r}(\lambda)$ is a matrix polynomial, which is called the right quotient, and $R_{r}(\lambda)$ is a matrix polynomial satisfying $\delta R_{r}(\lambda)<m$. The matrix polynomial $R_{r}(\lambda)$ is called the right remainder on division of $H(\lambda)$ by $X(\lambda)$. Similarly, we have

$$
H(\lambda)=X(\lambda) Q_{l}(\lambda)+R_{l}(\lambda)
$$

where $Q_{r}(\lambda)$ is the left quotient, and $R_{l}(\lambda)$ is the left remainder.

Lemma 1 (Lemma 3.1 in [20]). Suppose that matrix polynomials $A(\lambda)$ and $B(\lambda)$ are monic, $\delta A=m, \delta B=n$, $\delta C<m+n$. If the matrix polynomial equation,

$$
A(\lambda) X(\lambda)+Y(\lambda) B(\lambda)=C(\lambda),
$$

is solvable, then it has a solution $(X(\lambda), Y(\lambda))$ satisfying $\delta X<n, \delta Y<m$.

The following definitions about the Drazin inverse can be found in [21].

Definition 1. The smallest positive integer $k$ for which

$$
\operatorname{rank} A^{k}=\operatorname{rank} A^{k+1} \text {, }
$$

holds is called the index of $A$ and denoted by Ind $A$.

Definition 2. Let $A$ be a $r \times r$ matrix, $k=\operatorname{Ind} A$. If $X$ satisfies equations

$$
\begin{aligned}
A X & =X A, \\
A^{k+1} X & =A^{k}, \\
A X^{2} & =X,
\end{aligned}
$$

then $X$ is called the Drazin inverse of $A$ and denoted by $A_{d}$.

\section{Main Result}

We start with the following theorem about the special Sylvester matrix equation.

Theorem 1. Suppose $A, B \in \mathbb{C}^{r \times r}, n \geq k=\operatorname{Ind}(B)$. Then, the equation

$$
A X-X B=C B^{n},
$$

has a solution if and only if the equation

$$
A X-X B=C B^{k},
$$

has a solution.

Proof. Suppose equation (12) holds. Multiplying $B^{n-k}$ on the right side of equation (12), we have

$$
A\left(X B^{n-k}\right)-\left(X B^{n-k}\right) B=C B^{n} .
$$

This means equation (11) holds.

On the other hand, by the property of the Drazin inverse of matrix, there exists a matrix $B_{d}$ satisfying

$$
\begin{aligned}
B B_{d} & =B_{d} B, \\
B^{k} B_{d} & =B^{k-1} .
\end{aligned}
$$
have

Multiplying $\left(B_{d}\right)^{n-k}$ on the right side of equation (11), we

$$
A X\left(B_{d}\right)^{n-k}-X B\left(B_{d}\right)^{n-k}=C B^{n}\left(B_{d}\right)^{n-k}
$$

By equation (14), we have the following equation:

$$
A X\left(B_{d}\right)^{n-k}-X\left(B_{d}\right)^{n-k} B=C B^{k} .
$$

Thus, there exists a matrix $Y=X\left(B_{d}\right)^{n-k}$ that satisfies

$$
A Y-Y B=C B^{k} \text {. }
$$

The proof is completed.

With the help of Lemma 1 and Theorem 1, we can now prove the main result in this study.

Theorem 2. Suppose $A_{0}, B_{0} \in \mathbb{C}^{r \times r}$ are invertible matrices. If the Sylvester matrix equation

$$
\left(A_{1} \lambda+A_{0}\right) X(\lambda)+Y(\lambda)\left(B_{1} \lambda+B_{0}\right)=C_{0},
$$

has solutions, then it has low-degree solution $(L(\lambda), M(\lambda))$ satisfying

$$
\max \{\delta L(\lambda), \delta M(\lambda)\} \leq \min \left\{\operatorname{Ind}\left(A_{0}^{-1} A_{1}\right), \operatorname{Ind}\left(B_{1} B_{0}^{-1}\right)\right\}-1 .
$$

Proof. Suppose that the Sylvester matrix equation

$$
\left(A_{1} \lambda+A_{0}\right) X(\lambda)+Y(\lambda)\left(B_{1} \lambda+B_{0}\right)=C_{0},
$$

has a solution $(X(\lambda), Y(\lambda))$, where

$$
\begin{aligned}
& X(\lambda)=X_{n} \lambda^{n}+X_{n-1} \lambda^{n-1}+\cdots+X_{0}, \\
& Y(\lambda)=Y_{m} \lambda^{m}+Y_{m-1} \lambda^{m-1}+\cdots+Y_{0} .
\end{aligned}
$$

Then,

$$
\left(A_{1} \frac{1}{\lambda}+A_{0}\right) X\left(\frac{1}{\lambda}\right)+Y\left(\frac{1}{\lambda}\right)\left(B_{1} \frac{1}{\lambda}+B_{0}\right)=C_{0} .
$$


Without loss of generality, we may assume that $n \geq m$. Multiplying both sides of equation (22) by $\lambda^{n+1}$, left side of equation (22) by $A_{0}^{-1}$, and right side of equation (22) by $B_{0}^{-1}$, we have

$$
(A+I \lambda) \tilde{X}(\lambda)+\tilde{Y}(\lambda)(B+I \lambda)=C \lambda^{n+1},
$$

where $A=A_{0}^{-1} A_{1}, B=B_{1} B_{0}^{-1}, C=A_{0}^{-1} C_{0} B_{0}^{-1}$, and

$$
\begin{aligned}
& \tilde{X}(\lambda)=X_{n} B_{0}^{-1}+X_{n-1} B_{0}^{-1} \lambda+\cdots+X_{0} B_{0}^{-1} \lambda^{n}, \\
& \widetilde{Y}(\lambda)=A_{0}^{-1} Y_{m} \lambda^{n-m}+A_{0}^{-1} Y_{m-1} \lambda^{n-m+1}+\cdots+A_{0}^{-1} Y_{0} \lambda^{n} .
\end{aligned}
$$

By the division of matrix polynomials, we understand a representation in the form

$$
C \lambda^{n+1}=Q(\lambda)(I \lambda+B)+(-1)^{n+1} C B^{n+1},
$$

where $Q(\lambda)=C \lambda^{n}-C B \lambda^{n-1}+\cdots+(-1)^{n} C B^{n}$.

By substituting expression (25) into equation (23), it can be represented as

$$
(A+I \lambda) \tilde{X}(\lambda)+(\widetilde{Y}(\lambda)-Q(\lambda))(B+I \lambda)=(-1)^{n} C B^{n} .
$$

By Lemma 1, equation (26) being solved is equivalent to there exists $H, G \in \mathbb{C}^{r \times r}$ satisfying

$$
(A+I \lambda) H+G(B+I \lambda)=(-1)^{n} C B^{n},
$$

i.e.,

$$
A H+H \lambda+G B+G \lambda=(-1)^{n} C B^{n} .
$$

By comparing the coefficients of $\lambda$ on both sides of equation (28), we have $H+G=0$. Then, replacing $G$ by $-H$, equation (27) can be reduced to

$$
A H-H B=(-1)^{n} C B^{n} .
$$

By Theorem 1, there exists a matrix $D$, such that

$$
A D-D B=-C B^{k} \text {, }
$$

where $k=\operatorname{Ind} B=\operatorname{Ind}\left(B_{1} B_{0}^{-1}\right)$.

Let

$$
\begin{aligned}
L(\lambda) & =(-1)^{k-1} D B_{0} \lambda^{k-1}, \\
M(\lambda) & =A_{0}\left[(-1)^{k} D \lambda^{k-1}+(-1)^{k-1} C B^{k-1} \lambda^{k-1}+\cdots+(-1) \lambda C B+C\right] .
\end{aligned}
$$

Then, we have

$$
\begin{aligned}
& \left(A_{1} \lambda+A_{0}\right) L(\lambda)+M(\lambda)\left(B_{1} \lambda+B_{0}\right) \\
& =A_{0}(A \lambda+I) L(\lambda)+M(\lambda)(B \lambda+I) B_{0} \\
& =A_{0}\left[(-1)^{k-1} A D \lambda^{k}+(-1)^{k} D B \lambda^{k}+(-1)^{k-1} C B^{k} \lambda^{k}+C\right] B_{0} \\
& =(-1)^{k-1} A_{0}\left[A D-D B+C B^{k}\right] B_{0} \lambda^{k}+A_{0} C B_{0} \\
& =A_{0} C B_{0} \\
& =C_{0}, \\
\delta L(\lambda) \leq k-1 & =\operatorname{Ind}\left(B_{1} B_{0}^{-1}\right)-1, \\
\delta M(\lambda) \leq k-1 & =\operatorname{Ind}\left(B_{1} B_{0}^{-1}\right)-1 .
\end{aligned}
$$

Similarly, equation (18) has a solution $(L(\lambda), M(\lambda))$ satisfying

$$
\begin{gathered}
\delta L(\lambda) \leq \operatorname{Ind}\left(A_{0}^{-1} A_{1}\right)-1, \\
\delta M(\lambda) \leq \operatorname{Ind}\left(A_{0}^{-1} A_{1}\right)-1 .
\end{gathered}
$$

This completes the proof of Theorem 2.

We use the above theorem to calculate an example. This example also shows that the degree bound in Theorem 1 is the lowest one. 
Example 1. Consider equation

$$
\left(\left[\begin{array}{ccc}
-2 & 1 & 3 \\
-1 & 1 & 1 \\
1 & 0 & -2
\end{array}\right] \lambda+I_{3}\right) X(\lambda)+Y(\lambda)\left(\left[\begin{array}{ccc}
1 & -2 & 0 \\
1 & 0 & -1 \\
1 & -2 & 1
\end{array}\right] \lambda+I_{3}\right)=I_{3},
$$

where $I_{3}=\left[\begin{array}{lll}1 & 0 & 0 \\ 0 & 1 & 0 \\ 0 & 0 & 1\end{array}\right]$.

By Theorem 2 and

$$
\begin{aligned}
& \text { Ind }\left[\begin{array}{ccc}
-2 & 1 & 3 \\
-1 & 1 & 1 \\
1 & 0 & -2
\end{array}\right]=2, \\
& \text { Ind }\left[\begin{array}{ccc}
1 & -2 & 0 \\
1 & 0 & -1 \\
1 & -2 & 1
\end{array}\right]=2,
\end{aligned}
$$

we assume $\delta X=1$ and $\delta Y=1$, i.e.,

$$
\begin{aligned}
& X(\lambda)=X_{1} \lambda+X_{0}, \\
& Y(\lambda)=Y_{1} \lambda+Y_{0} .
\end{aligned}
$$

If we plug (36) into (34) and solve the equation, we obtain a solution

$$
\begin{aligned}
& X(\lambda)=\left[\begin{array}{ccc}
3 & -4 & -3 \\
1 & -2 & -1 \\
1 & 2 & -2
\end{array}\right] \lambda+\left[\begin{array}{ccc}
2 & 1 & -4 \\
0 & 0 & 0 \\
0 & 2 & -2
\end{array}\right], \\
& Y(\lambda)=\left[\begin{array}{ccc}
-1 & 2 & 1 \\
0 & 1 & 0 \\
0 & -1 & 0
\end{array}\right] \lambda+\left[\begin{array}{ccc}
-1 & 1 & 2 \\
0 & 1 & 0 \\
0 & 0 & -1
\end{array}\right] .
\end{aligned}
$$

Actually, there is no matrix polynomial with degree 0 satisfying equation (34).

\section{Data Availability}

No data, models, or codes were generated or used to support this study.

\section{Conflicts of Interest}

The authors declare that they have no conflicts of interest.

\section{Acknowledgments}

This work was supported by the Natural Science Foundation of Shandong Province, China (ZR2018PA002).

\section{References}

[1] S. D. Brierley and E. B. Lee, "Solution of the equation $A(z)$ $X(z)+X(z) B(z)=C(z)$ and its application to the stability of generalized linear systems," International Journal of Control, vol. 40, no. 6, pp. 1065-1075, 1984.
[2] E. Emre, "The polynomial equation $Q Q_{c}+R P_{c}=\Phi$ with application to dynamic feedback," SIAM Journal on Control and Optimization, vol. 18, no. 6, pp. 611-620, 1980.

[3] V. Kučera, "Algebraic approach to discrete stochastic control," Kybernetica, vol. 11, pp. 114-119, 1975.

[4] V. Kučera, Discrete Linear Control, Academia, Prague, Czech Republic, 1979.

[5] A. G. Wu, G. R. Duan, and Y. Xue, "Kronecker maps and Sylvester-polynomial matrix equations," IEEE Transactions on Automatic Control, vol. 52, no. 5, pp. 905-910, 2007.

[6] S. Zak, "On the polynomial matrix equation $A X+Y B=C$," IEEE Transactions on Automatic Control, vol. 30, no. 12, pp. 1240-1242, 1985.

[7] B. Zhou and G. R. Duan, "On the generalized Sylvester mapping and matrix equations," Systems \& Control Letters, vol. 57, no. 3, pp. 200-208, 2008.

[8] Y. Satake, M. Oozawa, T. Sogabe, Y. Miyatake, T. Kemmochi, and S. L. Zhang, "Relation between the T-congruence Sylvester equation and the generalized Sylvester equation," Applied Mathematics Letters, vol. 96, pp. 7-13, 2019.

[9] F. De Terán, B. Iannazzo, F. Poloni, and L. Robol, "Solvability and uniqueness criteria for generalized Sylvester-type equations," Linear Algebra and Its Applications, vol. 542, pp. 501-521, 2018.

[10] F. de Teran, B. Iannazzo, F. Poloni, and L. Robol, "Nonsingular systems of generalized Sylvester equations: an algorithmic approach," Numerical Linear Algebra with Applications, vol. 26, no. 5, 2019.

[11] Q. W. Wang, Z. H. He, and Y. Zhang, "Constrained two-sided coupled Sylvester-type quaternion matrix equations," Automatica, vol. 101, pp. 207-213, 2019.

[12] I. Gohberg, P. Lancaster, and L. Rodman, "Matrix polynomials, society for industrial and applied mathematics," 2009.

[13] H. K. Wimmer, "The generalized sylvester equation in polynomial matrices," IEEE Transactions on Automatic Control, vol. 41, no. 9, pp. 1372-1376, 1996.

[14] V. Kučera, "The matrix equation $A X+X B=C$," SIAM Journal of Applied Mathematics, vol. 26, pp. 15-25, 1974.

[15] I. Gohberg and L. Lerer, "Matrix generalizations of M. G. Krein theorems on orthogonal polynomials," Operator Theory: Advances and Applications, vol. 34, pp. 137-202, 1988.

[16] S. Barnett, "Regular polynomial matrices having relatively prime determinants," Mathematical Proceedings of the Cambridge Philosophical Society, vol. 65, no. 3, pp. 585-590, 1969.

[17] J. Feinstein and Y. Bar-Ness, "On the uniqueness of the minimal solution to the matrix polynomial equation $A(\lambda)$ $X(\lambda)+Y(\lambda) B(\lambda)=C(\lambda)$," Journal of the Franklin Institute, vol. 310, no. 2, pp. 131-134, 1980.

[18] M. A. Kaashoek and L. Lerer, "On a class of matrix polynomial equations," Linear Algebra and Its Applications, vol. 439, no. 3, pp. 613-620, 2013.

[19] V. M. Prokip, "About the uniqueness solution of the matrix polynomial equation $A(\lambda) X(\lambda)-Y(\lambda) B(\lambda)=C(\lambda)$," Lobachevskii Journal of Mathematics, vol. 29, no. 3, pp. 186-191, 2008.

[20] S. Chen and Y. Tian, "On solutions of generalized Sylvester equation in polynomial matrices," Journal of the Franklin Institute, vol. 351, no. 12, pp. 5376-5385, 2014.

[21] A. Ben-Israel and T. N. E. Greville, Generalized Inverses, Springer-Verlag, New York, NY, USA, 2003. 